Teknokultura. Revista de Cultura Digital y Movimientos Sociales

ISSNe: $1549-2230$

http://dx.doi.org/10.5209/TEKN.74287

\title{
Tecnologías digitales carismáticas, imaginario sociotécnico y ambivalencia: un estudio cualitativo sobre el uso de tablets por parte de personas mayores en Minas, Uruguay ${ }^{1}$
}

\author{
Alexander Castleton Flores ${ }^{2}$
}

Recibido: 12 de febrero de 2021 / Aceptado: 7 de abril de 2021 Open peer reviews

Resumen. Desde 2015 Uruguay ha desarrollado el Plan Ibirapitá, un programa que consiste en la entrega de tablets a personas mayores de bajos ingresos a quienes también se les ofrece capacitación. Este artículo enmarca este programa como un 'imaginario sociotécnico', basado en 'tecnologías carismáticas', y presenta un estudio cualitativo de cómo un grupo de personas mayores negocian la presencia de la tablet en sus vidas. Los datos fueron recogidos por medio de entrevistas semi-estructuradas en la ciudad de Minas (la capital del departamento de Lavalleja, el más envejecido del país) y analizados con codificación temática. La categoría central es 'ambivalencia generalizada', que está interrelacionada con otras categorías entre las que se incluyen 'los recursos de capacitación', 'la paciencia necesaria para operar la tablet', 'la falta de internet', 'las tablets se rompen y que requieren actualizaciones', y que 'las personas mayores entrevistadas llevan una vida ajetreada que incide en su uso'. De acuerdo con hallazgos empíricos y abordajes teóricos recientes, el artículo sugiere la posibilidad de que sea inadecuado presuponer necesidades tecnológicas preexistentes y abstractas de las personas mayores, y que estas sean identificadas y traducidas en su bienestar por medio de intervenciones tecnológicas carismáticas.

Palabras clave: adultos mayores; brecha digital; inclusión digital; tecnología digital.

\section{[en] Digital technologies, sociotechnical imaginary and ambivalence: a qualitative study on the use of tablets by older people in Minas, Uruguay}

\begin{abstract}
Since 2015 Uruguay has been developing Plan Ibirapitá-a program that consists of the delivery of tablets to low-income older people for free, who can also access training. This article frames this program as a 'sociotechnical imaginary', based on 'charismatic technologies', and presents a qualitative study of a group of how a group of older people negotiate the presence of the tablet in their lives. Data was gathered through semi-structured interviews in the city of Minas (the capital of Lavalleja Department, with the oldest population in the country) and analyzed through thematic analysis. The central category is 'generalized ambivalence', which interpenetrates with other categories. These other categories are related to 'training resources', a 'lack of patience to operate the tablet', ta 'lack of Internet', the fact that 'tablets broke down and required updates', and the notion that the busy lives of elderly people affect their use of the tablets. In accordance with recent empirical findings and theoretical approaches, the article suggests that it may be inappropriate to presuppose abstract, pre-existing technological needs of older people that can be identified and translated into their well-being through charismatic-technological interventions.
\end{abstract}

Keywords: digital gap; digital inclusion; digital technology; older adults.

Sumario. 1. Introducción. 2. Imaginario sociotécnico, carisma tecnológico y ambivalencia frente a la tecnología. 3. Metodología. 4. Resultados. 5. Conclusiones. 6. Referencias.

Cómo citar: Castleton Flores, A. (2021). Tecnologías digitales carismáticas, imaginario sociotécnico y ambivalencia: un estudio cualitativo sobre el uso de tablets por parte de personas mayores en Minas, Uruguay. Teknokultura. Revista de Cultura Digital y Movimientos Sociales, 18(2), 185-193. http://dx.doi.org/10.5209/TEKN.74287

\footnotetext{
Fuente de financiación: Consejo de Ciencias Sociales y Humanidades de Canadá.

MacEwan University (Canadá)

E-mail: castletona@macewan.ca; https://orcid.org/0000-0001-9191-3566 


\section{Introducción}

Uruguay es uno de los países más envejecidos de América (Brunet y Márquez, 2016). Desde el 2015 viene desarrollando el Plan Ibirapitá, un programa con el objetivo de incluir a la población mayor en la sociedad de la información mediante la entrega de tablets de forma gratuita. Actualmente se han distribuido más de 200.000 tablets.

Para obtener la tablet, la persona mayor debe recibir una jubilación de menos de aproximadamente 900 dólares americanos por mes. Además, hay capacitación disponible en todo el país en las oficinas del Ministerio de Educación y Cultura, y los beneficiarios del programa reciben $1 \mathrm{~GB}$ de Internet de forma gratuita. Sin embargo, la Quinta Encuesta de Uso del Plan Ibirapitá indica que aproximadamente un $60 \%$ de los que recibieron la $t a$ blet, no la usan (Plan Ibirapitá, 2019).

El Plan Ibirapitá podría enmarcarse en lo que Jasanoff (2015) define como un 'imaginario sociotécnico' basado en lo que Ames (2019) describe como 'tecnologías carismáticas'.

Muchas investigaciones que examinan el uso de la tecnología por parte de las personas mayores se basan en estudios a gran escala en los que la edad es un factor estudiado entre otras variables demográficas (QuaanHaase et al., 2016; Rogers, 2003). Estas investigaciones se centran en la llamada 'brecha gris', que sugiere que los factores relacionados con la edad (más allá de los ingresos, la educación y el género) dificultan la capacidad de las personas mayores para aprovechar las tecnologías digitales (Friemel, 2016). Una cuestión desatendida es, sin embargo, cómo se desarrollan los imaginarios sociotécnicos cuando «los individuos de carne y hueso se encuentran con la nueva tecnología y la incorporan a su vida diaria» (Hagberg, 2004, p. 165).

La investigación aquí presentada es cualitativa, aplicando entrevistas en la ciudad de Minas (la capital del Departamento de Lavalleja, el más envejecido del país), ubicada en la región centro-este de Uruguay. Las preguntas que orientaron esta investigación fueron: ¿Cómo se incorporan las tablets del Plan Ibirapitá en el tejido social preexistente de las personas mayores?', ‘¿cómo negocian estas la presencia de tal tecnología en su vida diaria?'

\section{Imaginario sociotécnico, carisma tecnológico y ambivalencia frente a la tecnología}

\subsection{El imaginario sociotécnico digital uruguayo y tablets carismáticas}

Jasanoff y Kim (2009, p. 121) desarrollaron el término ‘imaginario sociotécnico' para conceptualizar las «formas imaginadas colectivamente de vida y orden social reflejados en el diseño y cumplimiento de proyectos científicos y/o tecnológicos específicos de cada nación». Este concepto describe cómo la ciencia, la tecnología y la sociedad se unen en la práctica, permitiendo describir la co-creación de «órdenes políticos y proyectos tecnocientíficos» (McNeil et al., 2017, p. 449).
Bowman (2015) presenta un ejemplo de una aplicación concreta de este marco en su análisis de la implementación de las tecnologías de la información y la comunicación (TIC) en la Ruanda post-genocidio. Después de la guerra civil, a mediados de los noventa, el gobierno de Paul Kagame quiso dar una imagen de un «país moderno, tecnológicamente avanzado y de ingresos medios» (Bowman, 2015, p. 83), para lo que las TIC eran una parte fundamental del imaginario sociotécnico del país. Por ello, se presentaron como herramientas que «mejorarán la productividad y conducirán al crecimiento económico» y «mejorarán los resultados sociales y de desarrollo» (ibid., p. 91). Sin embargo, hubo problemas importantes a la hora de desarrollar este imaginario debido a cuestiones como la alfabetización, la infraestructura y los recursos económicos disponibles. El imaginario de una Ruanda moderna y desarrollada con las TIC tuvo que enfrentarse a la realidad empírica del país.

El contexto de Uruguay es ciertamente diferente al ruandés. Es considerado un país de altos ingresos por el Banco Mundial (2019), con un alto grado de alfabetización y una infraestructura sólida. Los gobiernos de izquierda que comenzaron en 2005 y se extendieron hasta 2019 enfatizaron la disminución de la brecha digital. Cuando el Dr. Tabaré Vázquez ganó las elecciones en el 2014, lanzó el Plan Ibirapitá en 2015 con la misión de «promover la inclusión digital de las personas mayores de bajos ingresos» (Memoria Anual, 2017, p. 1). El presidente Vázquez subrayó que el Plan Ibirapitá, más allá de otorgar tablets, aspiraba a que para los adultos mayores el envejecimiento pudiera ser «una etapa más de aprendizaje, actividad e integración en la vida social» (Uruguay Presidencia, 2016).

La tablet del Plan Ibirapitá aparece como una 'tecnología carismática' (Ames, 2019, p. 10), que, en las palabras de Vázquez (Uruguay Presidencia, 2016), «deriva su poder experimental y simbólicamente a través de la posibilidad o promesa de acción». Sin embargo, Ames (2019) explica que las tecnologías carismáticas son engañosas, ya que hacen que tanto la adopción tecnológica como el cambio social parezcan sencillos. Esto otorga a las tecnologías un cierto determinismo, donde el progreso tecnológico parece natural e incluso inevitable, enmarcado en un discurso transformador que elimina la agencia de los usuarios.

\subsection{Ambivalencia frente a nuevas tecnologías}

Diversos investigadores han tratado de comprender las relaciones concretas que las personas establecen con la tecnología a partir de distintos abordajes teóricos, como el de la apropiación de tecnología (Morales, 2018), las affordances (Hutchby, 2001), o el de la domesticación (Silverstone et al., 1999), entre otros. Un rasgo común en estos enfoques es que los objetos tecnológicos deben integrarse en los hábitos y quehaceres diarios de las personas mayores para que su uso sea significativo (p. ej., Quan Haase et al., 2016; Knowles y Hanson, 2018). Ignorar este acercamiento 'de abajo hacia arriba' conduce a usos matizados, al rechazo y al no uso, es decir, a ambivalencias (Peek et al., 2017; Givskov y Deuze, 2016). 
Desde los inicios de las ciencias sociales, la ambivalencia ha sido identificada como un aspecto capital de la vida social (Simmel, 1908), donde sentimientos contrapuestos «son el núcleo de nuestro ser, la materia de nuestra vida cotidiana. Son los cimientos de la sociedad» (Douglas, 1977, p. 51; ver Melnikov y Kotarba, 2016). Refiriéndose específicamente a la tecnología, Davis (2012, p. 958) define ambivalencia tecnológica como «la presencia simultánea de esperanzas utópicas y miedos distópicos». Rambe y Nel (2015), por su parte, explican que la ambivalencia frente a la tecnología:

Constituye una apreciación de la complejidad de las interacciones entre humanos y tecnología; de su carácter contextual, situado y contingente, que incluye los efectos múltiples y contradictorios de la tecnología en el mundo social donde se aplica (Rambe y Nel, 2015, p. 634).

En este sentido, en la medida que incrementa la mediatización tecnológica, la micro-resistencia por parte de las personas se hace cada vez más frecuente (Ribak y Rosenthal, 2015). Como señalan Chu y Yeo (2019, p. 151) «los usuarios, reconociendo tanto las promesas como las trampas de las tecnologías, aplican prácticas de desconexión en forma de micro-resistencia o preferencias a pequeña escala para negociar su experiencia mediatizada». Knowles y Hanson (2018), por ejemplo, subrayan que las elecciones de los adultos mayores son cruciales y que muchas veces su resistencia a la tecnología es una elección concienzuda basada en valores. Por lo tanto, muchos se resisten a algunas tecnologías y adoptan otras.

La noción de ambivalencia es interesante para matizar un acercamiento vertical como el Plan Ibirapitá y hace surgir la cuestión de cómo se relacionan, contextualmente, adultos mayores con las tablets entregadas de este modo a través de una política pública. Tal es el objetivo de este estudio.

\section{Metodología}

En un contexto donde casi el $60 \%$ de los que han recibido la tablet del Plan Ibirapitá dicen no utilizarla, el objetivo de esta investigación es hacer un examen exploratorio (ver Fernández-Ardèvol e Ivan, 2013) del significado de la tablet en la vida de algunas personas mayores en la ciudad de Minas y su posible adaptación a tejidos sociales preexistentes. En este sentido, el objetivo no es generalizar a ningún tipo de población, sino «comprender el mundo en el que [las personas] viven» donde «[d]esarrollan significados subjetivos dirigidos hacia ciertos objetos o cosas» (Creswell, 2013, p. 24), y así brindar insumos para la reflexión y futuras investigaciones (Flyvbjerg, 2005, pp. 39-40).

Minas, ubicada en la región centro-oriental de Uruguay, en el Departamento más envejecido, Lavalleja (Brunet y Márquez, 2016, p. 6), tiene 45.000 habitantes. Lavalleja cuenta con un $97 \%$ de cobertura LTE (Uruguay Presidencia, 2019, 11 mayo). Un contacto en la ciudad conectó al investigador con personas que habían recibido la tablet. También contactamos a otros participantes a través de la estrategia de 'bola de nieve', es decir, por la identificación por parte de los propios participantes de otras personas que pudieran ser entrevistadas (Creswell, 2007, p. 127).

De ese modo, se entrevistaron a 26 personas con edades comprendidas entre los 58 y 97 años y que antes de jubilarse desempeñaron distintas tareas como ser administrativas, rurales, comerciales, etc. La mayoría contaban con teléfonos celulares o inteligentes (excepto una persona que lo rechazaba concienzudamente), y aproximadamente un $75 \%$ tenían computadoras de escritorio o laptops (Tabla 1). El proceso de recolección de datos se concluyó cuando se alcanzó la saturación, es decir cuando los datos comenzaron a repetirse (Creswell, 2016. p. 110). Tanto la saturación temática como la saturación de datos guiaron este estudio, que refieren a la ejemplificación de temas identificados en los datos y el grado en que los nuevos datos repiten lo expresado en datos anteriores, respectivamente (Saunders et al., 2018).

Tabla 1. Características de los participantes. Fuente: elaboración propia

\begin{tabular}{|c|c|c|c|}
\hline \multicolumn{4}{|c|}{$\begin{array}{c}\text { 26 Participantes } \\
\text { Localidad: Minas, Uruguay (45.000 habitantes) }\end{array}$} \\
\hline Edades & Ocupaciones previas a la jubilación: & $\begin{array}{l}\text { Hace cuanto tienen la } \\
\text { tablet }\end{array}$ & Otras tecnologías \\
\hline $\begin{array}{l}\text { Rango } 58-97 \\
\text { Menos de } 65: 2 \\
\text { Entre } 65 \text { y } 70: 8 \\
\text { Entre } 71 \text { y } 80: 11 \\
\text { Entre } 81 \text { y } 90: 4 \\
\text { Más de 90: } 1\end{array}$ & $\begin{array}{l}\text { Servicios; empleados municipales y } \\
\text { estatales; maestros/as, trabajadores rurales. } \\
6 \text { participantes aún trabajaban 'bajo la mesa' } \\
\text { dando clases particulares de matemáticas, } \\
\text { como cuidadoras de personas más mayores, } \\
\text { en pequeños almacenes propios ubicados en } \\
\text { la propia casa. }\end{array}$ & 2 años & $\begin{array}{l}\text { Algunos tienen celular, } \\
\text { pero la mayoría } \\
\text { smartphone. } \\
3 / 4 \text { tienen PC o laptop. }\end{array}$ \\
\hline
\end{tabular}

Las entrevistas realizadas fueron semi-estructuradas y fueron transcritas textualment y codificadas temáticamente. Siguiendo las indicaciones de Creswell (2016), la codificación

implica determinar lo que se dice y asignar una etiqueta con un código a un pasaje de texto. Luego, agrupo códigos similares para construir evidencia de apoyo para categorías más amplias de información, llamadas temas. Estos temas a veces están interrelacionados para contar una historia y se convierten en los títulos de los hallazgos de un informe cualitativo (Creswell, 2016, p. 153). 
Partiendo de la teoría, el tema central fue la 'ambivalencia generalizada' y su relación con las siguientes categorías: 'recursos de capacitación', 'paciencia y falta de interés', 'tablets rotas', y 'vidas activas y ocupadas' (Figura 1). Estos temas son descritos a continuación.

Figura 1. Categoría central y relación con otras. Fuente: elaboración propia

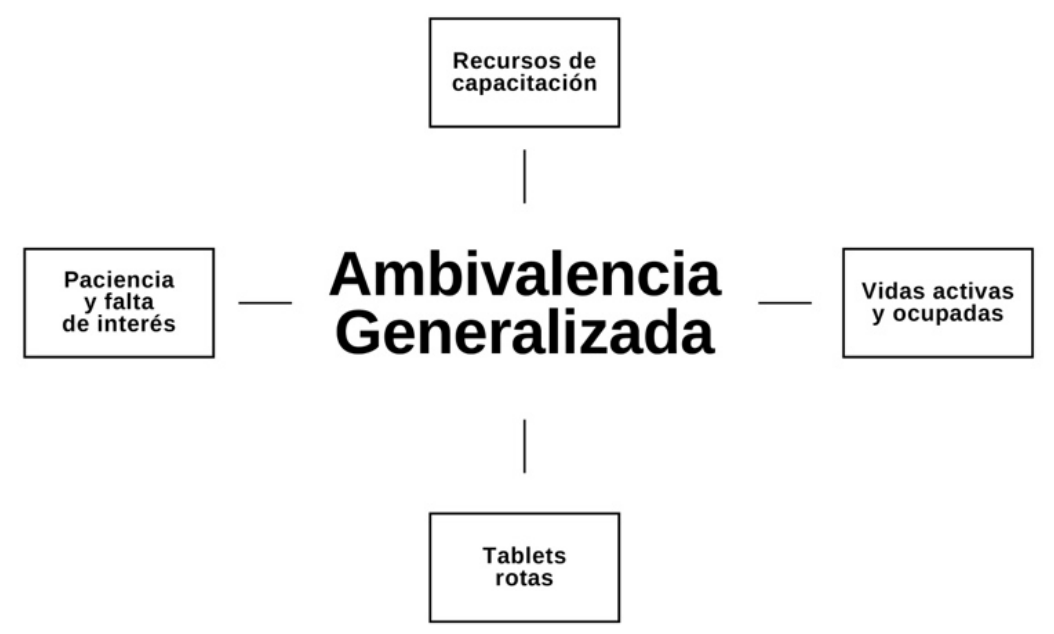

\section{Resultados}

\subsection{Ambivalencia generalizada}

Con el objetivo de comprender significados subjetivos, las preguntas formuladas a los participantes fueron generales (Creswell, 2007, p. 61), por ejemplo: ‘Ha cambiado su rutina con la tablet?' ¿Qué le gusta hacer con ella?'. Las primeras respuestas tendieron a ser positivas. Una de ellas, la de Raúl (68, trabajador rural jubilado que aún trabaja en su propia chacra), resume la percepción de los participantes:

Aunque hay una distancia de miles de kilómetros, podés comunicarte y ver muchas cosas que de otra manera serían imposibles. No puedo decir si mi vida es mejor; al menos es más práctica en muchos sentidos, principalmente debido a la comunicación (Raúl, E01).

Ese tipo de respuestas de carácter general eran constantes. Los participantes se refirieron a 'comunicación' y 'entretenimiento' cuando hablaban de la tablet, como las principales funciones que perciben en estos dispositivos digitales. Pero, al formular preguntas de sondeo, los participantes dijeron cosas como:

Sería lo mismo para mí si la tuviera [la tablet] o no. Investigador: ¿Siente que su vida ha mejorado con la tablet en algún sentido?

No. Mi vida es la misma (Gloria, 76, ex-trabajadora municipal, E02).

Investigador: ¿La tablet le permite estar más en contacto con lo que sucede en el mundo?

No sé... no lo creo (Raquel, 72, ex-maestra de escuela, E3).

Investigador: ¿Qué podría estimularlo a usar más la tablet?

No sé. No creo que pueda estar más interesado en ella. No más que ahora. A mí me es igual. Para mí [la tablet] no es esencial. Solo la uso como entretenimiento. Pero no es que yo diga, «me moriría si no la tuviera», no (Verdún, 65, trabajadora de cuidados, E04).

Para mi es lo mismo. Trabajo en mi chacrita y no dejo de trabajar por la tablet. Si tengo que trabajar, salgo a trabajar (Álvaro, ex-trabajador del sector rural, E05).

En gran medida, los participantes demostraron una posición ambivalente. Muchos de los participantes mencionaron algunos usos y cosas que les gusta hacer con la tablet generalmente con la ayuda de familiares o personas de confianza -algo que la literatura también ha señalado reiteradamente (p. ej. Hanninen et al., 2020)al mismo tiempo que presentan una postura crítica y de cierto rechazo a la tablet y otras tecnologías. Surgieron también otros elementos que alimentaban esta ambivalencia generalizada, que se describen a continuación (Figura 1).

\subsection{Recursos de capacitación}

Muchos de los/as participantes mencionaron que la red existente de recursos, como la formación en los centros de capacitación del Ministerio de Educación y Cultura no les satisfacía. Por ejemplo, Rodolfo (que trabajó en una distribuidora de bebidas), aunque parecía tener alguna intención de aprender, dijo que no había nada nuevo que le enseñaran en los centros de capacitación (cf. Vaportzis et al., 2017), por lo que se frustró y rápidamente perdió el interés:

Lo que pasa es que la estoy usando [la tablet] yo solo, $\mathrm{y}$ aún no lo he aprendido. No he tenido una clase para aprender correctamente. El otro día me dijeron que debía apuntarme y así lo hice, así que cuando fui, 
empezaron por explicar qué era la tablet. Yo ya lo sabía y quería aprender algo nuevo (Rodolfo, 71, E06).

A Rodolfo no le apetecía aprender las generalidades de la tablet: mencionó que quiere un uso más pragmático para cuestiones como la comunicación con los miembros de la familia-cuestión que apareció en casi todos los participantes que ven algún uso de la tablet o que tenían alguna opinión al respecto. Si bien las capacitaciones que se ofrecen tienen temas semanales como 'Email', 'Navegación' o 'Facebook' que podrían ser útiles, para Rodolfo existe una barrera infranqueable donde no quiere perder el tiempo en cosas que no le preocupan. Sara, quien trabajó en una cafetería antes de jubilarse, se hizo eco de esto:

El problema es que quizás las clases no brinden una instrucción completa de todo lo que nos interesa. Hay muchas cosas, no solo internet, sino también comunicación, hablar por teléfono, algunas llamadas que puedes hacer gratis (Sara, 69, E07).

Rodolfo y Sara parecen indicar que tal vez el mejor enfoque sería concentrarse en las necesidades concretas de las personas, donde la mejor táctica para involucrar a las personas mayores con la tecnología sería convencerlas del valor de alguna una aplicación específica que pueda ser de su interés, algo que la literatura ha señalado previamente (Kuoppamäki, 2018; Tatnall, 2014; Rogers, 2003). En este sentido, la capacitación en grupo podría ser un problema. Lucía, quien trabajó como cocinera, dijo al respecto:

Cuando te la dan [la tablet], te dicen «aquí sacás fotos, aquí hacés esto o lo otro»; pero salís de allí y no sabés nada. Te olvidás de todo (Lucía, 80, E08).

Otro problema para Jorge, quien fue pintor de construcción, fue que tiene una rutina establecida que incluye una siesta vespertina que le impide asistir a los entrenamientos y por lo tanto, perdió rápidamente interés para él. Dijo:

Cuando recibí la tablet, estaba muy emocionado; incluso quería ir a clase. Pero bueno, eran por la tarde. Al principio, esto era algo nuevo para mí, pero lo superé al instante (Jorge, 71, E09).

Si bien el caso de Jorge evidentemente no puede generalizarse, su actitud indica que no parece dispuesto a esforzarse para aprender (Vaportzis et al., 2017; Peek et al., 2017). Si las lecciones de tablet interfirieren con sus actividades establecidas, entonces, ¿por qué alterarlas cuando no hay mucho que valga la pena investigar en una tecnología?

\subsection{Paciencia y falta de interés}

La tablet también requiere algo que los participantes no suelen tener: paciencia. De hecho, un comentario común de los participantes fue que es lenta, por lo que pierden interés en ella: «Mirá, las tablets son lentas», se quejó VerKdún que aún trabaja como cuidadora de ancianos; mientras que Sara dijo, apuntando a la velocidad, «No son tablets que decís, iguau!». Sofía -hija de Violeta, una participante que trabajó como costureraafirmó: «Ella (Violeta) se pone ansiosa porque la tableta va lenta». Graciela, por ejemplo, describió esta falta de paciencia con la tablet como un defecto personal: «El problema es que la tableta es lenta y yo soy impaciente», también involucrando a miembros de su familia:

Mi nuera me dijo «el problema es que sos impaciente...» Tenés que esperar un rato; ella me dijo que esta tablet es lenta. Después de prenderla, funciona... Mi hijo le dijo a mi hija «dejala [a Graciela] porque se lo he explicado varias veces» (risas) (Graciela, 74, tuvo un pequeño comercio, E10).

Selva, quien fuera maestra de escuela, por otro lado, hizo una comparación con su teléfono celular:

[La tableta] se apaga repentinamente y no sabes qué hacer, o el internet se apaga. Es lento y hay que esperar. Quien toma mate, probablemente tomaría tres mientras espera que aparezcan las cosas... ¿Por qué esperar tanto si el teléfono es instantáneo? (Selva, 67, ex-maestra, E11).

El punto de Selva es válido. Si el uso principal de las TIC es la comunicación, como insistieron la mayoría de los/as participantes, quizás una tablet no sea el mejor medio. Más aún en el caso de países como Uruguay que han experimentado el auge de WhatsApp como una forma principal de comunicación (El País, 2018).

En su estudio seminal sobre la difusión de innovaciones, Rogers (2003) señaló como cruciales la ventaja relativa de una tecnología frente a otras, así como la compatibilidad con lo que se busca de ellas. Los teléfonos celulares o teléfonos inteligentes suelen ser mucho más efectivos en lo que respecta a la comunicación y, al igual que en muchos países latinos (ver Fernández-Ardévol y Rosales, 2018). Los participantes también destacaron que no tiene sentido usar la tablet sin acceso a internet y, reafirmando la ambivalencia generalizada, solían decir que el internet que reciben gratis con el programa (1 GB que se puede recargar por una tarifa) no es suficiente. Esto significa que algunas personas mayores realmente intentan usar o quieren usar la tableta, y la falta de uso proviene de un tipo diferente de frustración, como comentó el extrabajador municipal Humberto:

El giga que te dan no me alcanza. Esto es un mal negocio porque de esa manera obligan a las personas mayores a inscribirse en un programa mensual. Algunos jubilados como yo no llegan a los 20.000 pesos, ¿cómo pueden pagar 900 por internet? Eso no puede ser. ¿Por qué regalarías algo que generaría eso? Es por eso que tanta gente simplemente la guardó [a la tablet] (Humberto, 73, E12).

\subsection{Tablets rotas y actualizaciones requeridas}

Otro problema ampliamente reiterado fue que las tablets se rompían fácilmente o necesitaban actualizaciones. 
Por ejemplo, requieren que las personas mayores tengan cuidado con el cargador, lo que no siempre es el caso, y fue una queja frecuente. Es común que las tablets acaben siendo enviadas a Montevideo para ser reparadas, lo que suele desanimar aún más su uso porque lleva tiempo y cuesta 500 pesos. Así lo apreciamos en el siguiente extracto de entrevista:

Gloria: Esta rota, no funciona, no puedo usarla.

Investigador: ¿La envió a Montevideo para que la arreglaran?

Gloria: No, porque la mandó mi amiga y le costó 500 pesos. Y dice que no recibió la misma tableta porque se borraron todos sus contactos... todas las imágenes. Es por eso que no quiero enviarla cuando está rota porque tengo algunas lindas fotos con mi nieto. Si todo se borra, prefiero no enviar eso (Gloria, E02).

Otros participantes como Gladys, quien trabajó como empleada doméstica, fueron más concisos en sus respuestas señalando los problemas y las confusiones que traen las actualizaciones:

Ahora [la tablet] no funciona muy bien porque aparece un mensaje que dice: "Aplicación de Google detenida». No sé lo que está mal. Tengo que ir y arreglarlay ver qué tiene de malo. Apenas escribo dos letras y aparece ese mensaje. Lo que me vuelve loca es que hay que actualizarla constantemente. No quiero estar encima de eso, pero cuando [mi sobrino] viene, la actualiza, me da consejos y yo puedo hacer preguntas (Gladys, 62, E13).

El hecho de que las tablets se descompongan y necesiten actualizaciones se sumó a la falta de paciencia de los/as participantes y la falta de utilidad percibida de las sesiones de formación, lo que dificulta todo ello su uso por parte de las personas mayores.

\subsection{Vidas activas y ocupadas}

En la última década, se ha hecho hincapié alrededor del mundo sobre el envejecimiento activo (Lassen, 2017). Sin embargo, hubo bastante consenso entre los participantes en que entendían sus vidas ocupadas como están, y la jubilación no significa necesariamente tiempo libre para ellos. Repasemos lo que dijeron algunos de ellos:

Si pasara más tiempo en casa, la usaría más [la tablet]. Pero tengo otras actividades que me llevan mucho tiempo, así que la uso siempre que tengo tiempo libre o para algún tema específico (Washington, E14).

Investigador: ¿[la tablet] le quita tiempo?]

No, porque siempre estoy ocupada. Tengo que trabajar en mis proyectos, porque voy a un taller. Pinté durante muchos años; ya no pinto, pero voy a clases de marquetería y actualmente estoy haciendo un dibujo. Entonces, si estoy haciendo una imagen, eso es lo primero. O si estoy tejiendo, eso es lo que estoy haciendo (Sara, E07).

No uso mucho [la tablet]. Tengo una laptop, pero no la uso mucho. De vez en cuando recibo correos electró- nicos, los leo y respondo. Pero no me gusta mucho la tecnología, soy de otra época... No es que no me guste. Hago otras cosas: tejo, hago artesanías en madera... A veces la agarro para jugar al solitario, para tomar alguna foto que me guste si la tengo a mano (Raquel, E15).

Lo que se desprende de aquí es que es quizás necesario escrutar las visiones abstractas de lo que se entiende por 'envejecimiento activo' y el rol de las tecnologías digitales (Casado-Muñoz, 2015). En investigaciones futuras será necesario examinar lo que significa estar activo y ocupado para las personas mayores, y el rol de los diversos dispositivos en sus vidas ocupadas.

\section{Conclusiones}

Los participantes en este estudio expresaron ambivalencia frente a la tablet. Por un lado, percibían potencialidades de un dispositivo que equivale a comunicación y entretenimiento, y destacaron la necesidad de estar conectados para estar al día. Por otro lado, cuestionaron la importancia de la tecnología en su vida cotidiana dado que frecuentemente optaban por dejar de lado los dispositivos.

Además de percibir potencialidades relacionadas al entretenimiento y comunicación, los participantes de esta investigación percibieron que los recursos de capacitación no cumplían con sus necesidades, que les faltaba la paciencia requerida. Se quejaron de la falta de acceso a Internet, que las tablets se estropeaban y que requerían actualizaciones, y afirmaron que viven vidas suficientemente ocupadas. Por tanto, la noción de ambivalencia captura el contradictorio e incluso desconcertante discurso de los participantes, que fluctuaba entre la utilidad de la tecnología, su falta de uso o necesidad, y los problemas que trae. La ambivalencia, entonces, aparece como la principal categoría a través de la que podemos comprender cómo los uruguayos mayores negocian constantemente cómo incorporar las tablets del Plan Ibirapitá y otras tecnologías digitales en sus vidas.

Este estudio-sin ser una evaluación de política-muestra que, en la medida en que la vida cotidiana es compleja y generalmente ambivalente, las formas en que las personas llevan un artefacto a su vida privada, dándole forma y construyendo significados, también lo son (Nimrod, 2016; Pirhonen et al., 2020; Quan Haase et al., 2016; KaniaLundholm, 2019; Gallistl et al., 2020; Peek et al., 2017; Lagerkvist, 2017). Lo cierto es que un $60 \%$ de los que han recibido la tablet dicen no utilizarla (Plan Ibirapitá, 2019), y quizás sea necesario considerar la posibilidad de que no es adecuado presuponer necesidades tecnológicas preexistentes de personas mayores de forma abstracta, $y$ que estas necesidades pueden ser identificadas, mapeadas y traducidas a su bienestar por medio de intervenciones tecnológicas carismáticas. En este sentido, esta investigación coincide con hallazgos empíricos y discusiones teóricas recientes que critican políticas verticales basadas en intervenciones a gran escala que apuntan a la adopción de tecnologías (Cozza, Östlund y Peine, 2021; Peine y Neven, 2020; Ames, 2019). 
De los hallazgos de este estudio cualitativo, se podrían definir algunos enunciados que pueden servir como hipótesis para futuras investigaciones (Creswell, 2007), o incluso para la transformación de esta investigación exploratoria en una longitudinal-ello teniendo en cuenta el hecho global de la pandemia del covid-19, durante la cual se ha enfatizado el uso de tecnologías digitales por parte de adultos mayores (Etzioni, 2020; Seifert, 2020). A continuación, se formulan cuatro enunciados con correspondientes preguntas de investigación:

1. La ambivalencia frente a las tecnologías digitales hace que las personas mayores practiquen su capacidad de agencia y decidan activamente el rol de las tecnologías digitales en su vida (cf. Hanson y Knowles, 2018; Quan-Haase et al., 2016). Dadas las medidas sanitarias de distanciamiento físico tomadas en Uruguay y el resto del mundo durante la pandemia: ¿de qué formas la experiencia durante el covid-19 cambió la relación de los adultos mayores con las tecnologías digitales? ¿Cómo ha variado la ambivalencia?

2. Los adultos mayores tienden a rechazar capacitaciones generales que no atienden directamente a los usos inmediatos que ellos ven en las tecnologías y con las que no se sienten cómodos (cf. Hunsaker et al., 2020; Olsson y Viscovi, 2018; Tatnall, 2014): ¿de qué maneras cambió la pandemia las formas en que los adultos mayores aprenden sobre tecnologías?

3. La impaciencia y la autopercepción de vivir vidas ocupadas son factores que impactan en la relación que los adultos mayores establecen con las tecnologías: ¿cuál ha sido el cambio traído por la situación de distanciamiento físico y cierres de emergencia en cuanto a las percepciones de impaciencia o estar ocupado? ¿Se ha traducido esto en un mayor uso de tecnologías digitales?

4. Las tablets del Plan Ibirapitá presentan algunos inconvenientes relacionados tanto al hardware (se estropean) como al software (requieren actualizaciones y la falta de internet), lo que lleva a que muchas veces se dejen de lado: ¿cómo ha influido la pandemia en el mayor uso que hacen los adultos mayores de las tecnologías digitales e Internet? ¿Cuáles son los mecanismos detrás de estos procesos sociales?

Para terminar, es necesario remarcar algunas limitaciones de este estudio. Dado su carácter cualitativo, tiene la limitación de que los resultados no pueden generalizarse, lo que podría afectar su transferibilidad (Yilmaz, 2013). En cualquier caso, su objetivo no era extrapolar desde una ciudad de Uruguay, sino comprender el mundo en el que viven y desarrollan significados las personas mayores (Creswell, 2007), y aportar insumos para futuras investigaciones (Flyvbjerg, 2005). Por lo tanto, aunque los hallazgos de este estudio y las recomendaciones de investigación adicional son limitados, podrían ser en todo caso útiles para países que aún no han introducido ni desarrollado políticas tecnológicas verticales para los adultos mayores. Por otro lado, para mejorar aún más la credibilidad de una investigación cualitativa como esta (Yilmaz, 2013) -aunque las categorías temáticas en este estudio estaban saturadas (Saunders et al., 2018)- los proyectos futuros tendrían que incluir un muestreo intencional más preciso de acuerdo con variables definidas de antemano, permitiendo así comparaciones.

\section{Referencias}

Ames, M. G. (2019). The Charisma Machine: The Life, Death, and Legacy of One Laptop per Child. Massachusetts: MIT Press. Banco Mundial (2019, 9 de abril). Uruguay Overview. Recuperado de: https:/www.worldbank.org/en/country/uruguay/overview Bowman, M. W. (2015). Imagining a modern Rwanda: sociotechnological imaginaries. En Dreamscapes of Modernity: Sociotechnical Imaginaries and the Fabrication of Power (S. Jasanoff y S.H. Kim, pp. 79-102). Chicago: University of Chicago Press.

Brunet, N. y Márquez, C. (2016). Envejecimiento y personas mayores en Uruguay. En Atlas sociodemográfico y de la desigualdad en Uruguay, Fascículo 7 (J.J. Calvo, pp. 1-79). INE: Uruguay.

Casado-Muñoz, R., Lezcano, F. y Rodríguez-Conde, M. J. (2015). Envejecimiento activo y acceso a las tecnologías: Un estudio empírico evolutivo. Comunicar 22(45), 37-46. http://dx.doi.org/10.3916/C45-2015-04

Caspi, A., Daniel, M. y Kavé, G. (2019). Technology makes older adults feel older. Aging \& mental health 23(8), 1025-1030. https://doi.org/10.1080/13607863.2018.1479834

Creswell, J.W. (2016). 30 Essential Skills for the Qualitative Researcher. Londres: Sage.

Creswell, J.W. (2013). Qualitative Inquiry \& Research Design: Choosing among Five Approaches, 3rd edition. Londres: Sage.

Creswell, J.W. (2007). Qualitative Inquiry \& Research design: Choosing among five approaches, 2nd edition. Londres: Sage.

Cozza, M., Östlund, B. y Peine, A. (2021). When theory meets practice in entanglements of ageing and technology. TECNOSCIENZA: Italian Journal of Science \& Technology Studies, 11(2), 5-12.

Davis, J. L. (2012). Social media and experiential ambivalence. Future Internet, 4(4), 955-970. https://doi.org/10.3390/fi4040955

Decreto 130/015 (2015, 25 de mayo). Creación del Programa Ibirapitá. Recuperado de: https://www.impo.com.uy/bases/ decretos/130-2015

Douglas, J. (1977). Existential sociology. En Existential Sociology (Jack D. Douglas y J. M. Johnson, pp. 3-73). Nueva York: Cambridge University Press.

El País (2018, 29 de noviembre). Uruguayos en internet: cuál red social es la estrella y cuántos grupos de WhatsApp tienen. Vida Actual. Recuperado de: https://www.elpais.com.uy/vida-actual/uruguayos-internet-red-social-estrella-cuantos-gruposwhatsapp.html 
Etzioni, A. (2020). The Sociology of Surviving the Coronavirus. The National Interest. Recuperado de: https://nationalinterest. org/feature/sociology-surviving-coronavirus-133512

Fernández-Ardèvol, M. e Ivan, L. (2013). Older people and mobile communication in two European contexts. Romanian Journal of Communication \& Public Relations, 15(3), 83-98. https://doi.org/10.21018/rjcpr.2013.3.196

Flyvbjerg, B. (2005). Social science that matters. Foresight Europe 2, 38-42.

Friemel, T. N. (2016). The digital divide has grown old: determinants of a digital divide among seniors. New Media \& Society, 18(2), 313-331. http://dx.doi.org/10.1177/1461444814538648

Gallistl, V., Rohner, R., Seifert, A. y Wanka, A. (2020). Configuring the older non-user: Between research, policy and practice of digital exclusion. Social Inclusion, 8(2), 233-243. http://dx.doi.org/10.17645/si.v8i2.2607

Givskov, C. y Deuze, M. (2018). Researching new media and social diversity in later life. New Media \& Society, 20(1), $399-412$. https://doi.org/10.1177/1461444816663949

Hagberg, J.-E. (2004). Old people, new and old artefacts: Technology for later life. En Changing Worlds and the Ageing Subject: Dimensions in The Study of Ageing and Later Life (B.M. Öberg, A.L. Närvänen, E. Näsman y E. Olson, pp. 161-184). Aldershot: Ashgate.

Hänninen, R., Taipale, S. y Luostari, R. (2020). Exploring heterogeneous ICT use among older adults: The warm experts' perspective. New Media \& Society. Publicación online adelantada. https://doi.org/10.1177/1461444820917353

Hunsaker, A. Nguyen, M.H. Fuchs, J. Djukaric, T. Hugentobler, L. y Hargittai E (2019). "He explained it to me and i also did it myself': how older adults get support with their technology uses. Socius, 5, 1-13. https://doi.org/10.1177/2378023119887866

Hutchby, I. (2001). Technologies, texts and affordances. Sociology, 35(2), 441-456. https://doi.org/10.1177/S0038038501000219

Jasanoff, S. y Kim, S.H. (2009). Containing the atom: sociotechnical imaginaries and nuclear power in the United States and South Korea. Minerva, 47, 119-146. https://doi.org/10.1007/s11024-009-9124-4

Jasanoff, S. (2015). Future imperfect: science, technology, and the imaginations of modernity. En Dreamscapes of Modernity: Sociotechnical Imaginaries and the Fabrication of Power (S. Jasanoff y S.H. Kim, pp. 1-33). Chicago: University of Chicago Press.

Kania-Lundholm, M. (2019). Slow side of the divide? Older ict non-and seldom-users discussing social acceleration and social change. Digital Culture \& Society, 5(1), 85-104. https://doi.org/10.14361/dcs-2019-0106

Knowles, B. y Hanson, V. L. (2018). The wisdom of older technology (non) users. Communications of the ACM, 61(3), $72-77$. https://doi.org/10.1145/3179995

Kuoppamäki, S. (2018). The role of age and life course stage in digital consumption. (Tesis doctoral, Universidad de Jyväskylä). Recuperado de: https://jyx.jyu.fi/handle/123456789/60180

Lagerkvist, A. (2017). Existential media: toward a theorization of digital thrownness. New Media \& Society, 19(1), 96-110. https://doi.org/10.1177/1461444816649921

Lassen, A. J. (2017). Shaping old age: innovation partnerships, senior centres and billiards tables as active ageing technologies. En Framing Age: Contested Knowledge in Science and Politics (B. Loffeier Majerus y T. Moulaert, pp. 222-236). Londres: Routledge.

Melnikov, A. y Kotarba, J. (2016). Existential Sociology. En The Blackwell Encyclopedia of Sociology (G. Ritzer, pp. 1-6). Oxford: Blackwell Publishing.

McNeil, M., M. Arribas-Ayllon, J. Haran, A. Mackenzie, y Tutton, R. (2017). Conceptualizing imaginaries of science, technology, and society. En The Handbook of Science and Technology Studies, 4th ed. (U. Felt, R. Fouche, C. A. Miller, y L. Smith-Doeer, pp. 435-463). Cambridge: MIT Press.

Memoria Annual (2017). Plan Ibirapitá. Recuperado de: https://ibirapita.org.uy/wp-content/uploads/2018/04/plan_ibirapita_ MEMORIA-2017.pd

Morales, S. (2018). La apropiación de tecnologías. Ideas para un paradigma en construcción. En Acerca de la apropiación de tecnologías: teoría, estudios y debates (S. Lago Martínez; A. Álvarez; M. Gendler y A. Méndez, pp. 23-34). Buenos Aires: Ediciones del Gato Gris.

Nimrod, G. (2016). The hierarchy of mobile phone incorporation among older users. Mobile Media \& Communication, 4(2), 149168. https://doi.org/10.1177/2050157915617336

Olsson, T. y Viscovi, D. (2018). Warm experts for elderly users: who are they and what do they do? Human Technology, 14(3), 324-342. https://doi.org/10.17011/ht/urn.201811224836

Peek, S. T. M., Luijkx, K. G., Vrijhoef, H. J. M., Nieboer, M. E., Aarts, S., Van der Voort, C. S., M.D. Rijnaard y Wouters, E. J. M. (2017). Origins and consequences of technology acquirement by independent-living seniors: towards an integrative model. BMC Geriatrics 17(1), 189. https://doi.org/10.1186/s12877-017-0582-5

Peine, A. y Neven, L. (2018). From intervention to co-constitution: new directions in theorizing about aging and technology. The Gerontologist, 59(1), 15-21. https://doi.org/10.1093/geront/gny050

Peine, A. y Neven, L. (2020). The co-constitution of ageing and technology-a model and agenda. Ageing \& Society, Publicación online adelantada. https://doi.org/10.1017/S0144686X20000641

Pirhonen, J., Lolich, L., Tuominen, K., Jolanki, O. y Timonen, V (2020). 'These devices have not been made for older people's needs'-Older adults' perceptions of digital technologies in Finland and Ireland. Technology in Society, 62, 101287. https:// doi.org/10.1016/j.techsoc.2020.101287

Plan Ibirapitá (2019). Quinta Encuesta de Uso. Departamento de Evaluación y Monitoreo. Recuperado de: https://ibirapita.org. uy/wp-content/uploads/2019/10/Ibirapit\%C3\%A1-encuesta-de-uso-2019.pdf

Ribak, R. y Rosenthal, M. (2015). Smartphone resistance as media ambivalence. First Monday, 20(11). http://dx.doi.org/10.5210/ fm.v20i11.6307

Rogers, E. M. (2003). Diffusion of innovations, 5th ed. Nueva York: Free Press.

Saunders, B., Sim, J., Kingstone, T., Baker, S., Waterfield, J., Bartlam, B., Burroughs, H. y Jinks, C. (2018). Saturation in qualitative research: exploring its conceptualization and operationalization. Quality \& Quantity, 52(4), 1893-1907. https:// doi.org/10.1007/s11135-017-0574-8 
Seifert, A. (2020). The digital exclusion of older adults during the COVID-19 pandemic. Journal of Gerontological Social Work, 63(6-7), 674-676. https://doi.org/10.1080/01634372.2020.1764687

Silverstone, R., Hirsch, E. y D. Morley (1999). Information and communication technologies and the moral economy of the household. En Consuming Technologies: Media and In-formation in Domestic Spaces (R. Silverstone y E. Hirsch, pp. 13-28). Londres: Routledge.

Simmel, G. (1908). Soziologie. Leipzig: Duncker und Humboldt.

Tatnall, A. (2014). ICT, education and older people in Australia: A socio-technical analysis. Education and Information Technologies, 19(3), 549-564. https://doi.org/10.1007/s10639-013-9300-x

Uruguay Presidencia (2019, 11 de mayo). Uruguay alcanzará el 100 \% de cobertura en tecnología de fibra óptica. Presidencia de la República. Recuperado de: https://www.presidencia.gub.uy/comunicacion/comunicacionnoticias/telecomunicacionesy-energias-renovables

Uruguay Presidencia (2019, 1 de marzo). Presidente Vázquez en el Antel Arena. Estamos cambiando la centralidad histórica del país, brindando a los gurises oportunidades inexistentes hace 15 años. Presidencia de la República. Recuperado de: https:// www.presidencia.gub.uy/comunicacion/comunicacionnoticias/vazquez-antel-arena-descentralizacion-educacion-gobiernodigital-plan-ceibal-ibirapita

Uruguay Presidencia (2016, 9 de julio). Plan Ceibal es una revolución irreversible y un gran avance hacia acceso igualitario al conocimiento. Presidencia de la República. Recuperado de: https://www.presidencia.gub.uy/comunicacion/ comunicacionnoticias/vazquez-plan-ceibal-foro-ciencia-unesco

Vaportzis, E., Giatsi Clausen, M. y Gow, A. J. (2017). Older adults' perceptions of technology and barriers to interacting with tablet computers: a focus group study. Frontiers in Psychology, 8. https://doi.org/10.3389/fpsyg.2017.01687

Yilmaz, K. (2013). Comparison of quantitative and qualitative research traditions: Epistemological, theoretical, and methodological differences. European Journal of Education, 48(2), 311-325. https://doi.org/10.1111/ejed.12014 